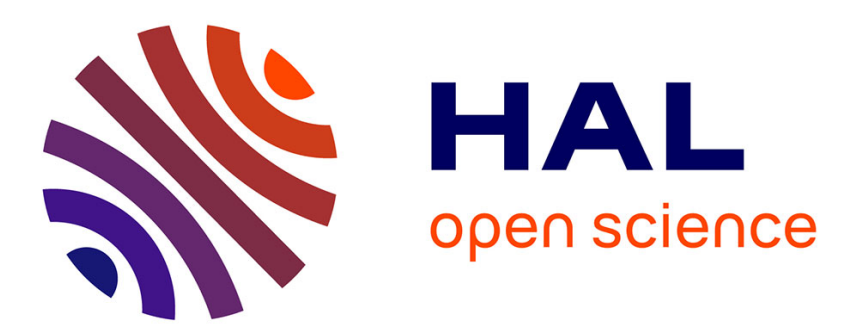

\title{
Numerical and experimental investigations of vector soliton bound-states in a Kerr planar waveguide
}

\author{
M. Delque, M. Chauvet, H. Maillotte, T. Sylvestre
}

\section{To cite this version:}

M. Delque, M. Chauvet, H. Maillotte, T. Sylvestre. Numerical and experimental investigations of vector soliton bound-states in a Kerr planar waveguide. Optics Communications, 2005, 249, $\mathrm{n}^{\circ} 1-3$, pp.285-291. hal-00097378

\section{HAL Id: hal-00097378 \\ https://hal.science/hal-00097378}

Submitted on 28 Aug 2013

HAL is a multi-disciplinary open access archive for the deposit and dissemination of scientific research documents, whether they are published or not. The documents may come from teaching and research institutions in France or abroad, or from public or private research centers.
L'archive ouverte pluridisciplinaire HAL, est destinée au dépôt et à la diffusion de documents scientifiques de niveau recherche, publiés ou non, émanant des établissements d'enseignement et de recherche français ou étrangers, des laboratoires publics ou privés. 


\title{
Numerical and experimental investigations of vector soliton bound-states in a Kerr planar waveguide
}

\author{
Michaël Delqué *, Mathieu Chauvet, Hervé Maillotte, Thibaut Sylvestre \\ Département doptique P.M. Duffieux, Institut FEMTO-ST, Université de Franche-Comté, Centre National de la Recherche \\ Scientifique UMR 6174, route de Gray, F-25030 Besançon Cedex, France
}

Received 21 October 2004; received in revised form 17 December 2004; accepted 18 December 2004

\begin{abstract}
We investigate both numerically and experimentally the stability of a vector three-soliton bound state in a Kerr $\mathrm{CS}_{2}$ planar waveguide using the circular polarizations as the two-component of the multimode vector soliton (MVS). The molecular-reorientation-induced Kerr nonlinearity of $\mathrm{CS}_{2}$ leads in this case to a strength of cross-phase modulation that is 7 times larger than that of self-phase modulation. We demonstrate that, under these conditions, the MVS exhibits symmetry-breaking instability leading to different output intensity patterns ranging from three to one-hump vector solitons.
\end{abstract}

(C) 2004 Elsevier B.V. All rights reserved.

Keywords: Spatial soliton; Vector soliton; Multimode soliton; Symmetry breaking instability

\section{Introduction}

Among the wide variety of spatial optical solitons, multimode vector solitons (MVSs) are of growing interest due to their intriguing dynamical properties and their potential applications for future all-optical technologies. MVS consist of

\footnotetext{
* Corresponding author. Tel.: +33 3816664 26; fax: +33 3 81666423.

E-mail address: michael.delque@univ-fcomte.fr (M. Delqué).
}

multiple symmetric and anti-symmetric polarization components that trap each other through crossphase modulation (XPM) in a nonlinear medium [1-3], which constitutes in this way a light-induced symmetric multimode waveguide. The stability of such vector soliton bound-states results from a precise balance between the self-focusing nature of symmetric components and the diffraction of their anti-symmetric counterparts. MVS have recently been found in bulk photorefractive media under the form of the so-called multihump [4,5] or dipole-mode vector solitons [6,7]. Note however that 
the dark-bright soliton pairs observed earlier in $1 \mathrm{D}$ domain can be viewed as a MVS since the dark soliton is the first higher-order mode of its induced waveguide at cutoff [8]. In a pure Kerr medium, they were also demonstrated in a $(1+1 \mathrm{D})$ config- not only to study their stability, but also to assess the various parameters that initiate SBI. The method consists in solving numerically the following system of coupled nonlinear Schrödinger equations (CNLSE):

$$
\left\{\begin{array}{l}
\frac{\partial E_{x}}{\partial z}=\frac{i}{2 k_{x}} \frac{\partial^{2} E_{x}}{\partial x^{2}}+\mathrm{i} \gamma\left[\left(\left|E_{x}\right|^{2}+(1-B)\left|E_{y}\right|^{2}\right) E_{x}+B E_{y}^{2} E_{x}^{*} \mathrm{e}^{-2 i \Delta k z}\right] \\
\frac{\partial E_{y}}{\partial z}=\frac{i}{2 k_{y}} \frac{\partial^{2} E_{y}}{\partial x^{2}}+\mathrm{i} \gamma\left[\left(\left|E_{y}\right|^{2}+(1-B)\left|E_{x}\right|^{2}\right) E_{y}+B E_{x}^{2} E_{y}^{*} \mathrm{e}^{+2 i \Delta k z}\right]
\end{array}\right.
$$

uration, i.e., in a planar waveguide geometry [9]. In the latter case, it has been reported that the bimodal vector soliton made by two incoherently coupled circular polarizations undergoes a sharp symmetry-breaking instability (SBI) [9]. This phenomenon has been theoretically studied in saturable medium [10]. Such an instability, that induces sudden transfer of the energy from one mode to the other, could be advantageously applied to spatial optical switching as the light remains confined, after the breaking, into a fundamental soliton. This elliptically polarized fundamental soliton and its stability has been widely studied [11-15]. However, the experimental demonstration previously reported on MVS in [9] was restricted to the case of the first member of the MVS's family and it is of particular interest to know how SBI can affect the propagation dynamics of higher-order MVS. In this paper, we investigate both numerically and experimentally the nonlinear propagation of a three-hump MVS propagating in a slab planar waveguide filled with carbon disulfide $\left(\mathrm{CS}_{2}\right)$ as a Kerr medium. The molecular-reorientationinduced Kerr nonlinearity of $\mathrm{CS}_{2}$ leads in this case to a strength of XPM that is 7 times larger than self-phase modulation (SPM). Under these conditions, several different output intensity patterns ranging from soliton bound-states (stable propagation) to two or single-hump intensity distribution (unstable behavior) due to SBI are observed.

\section{Numerical investigations}

The numerical analysis of the nonlinear propagation of MVS in a Kerr planar waveguide allows
Here $z$ is the propagation direction coordinate and $x$ the free transverse dimension of the planar waveguide. $E_{x}(x, z)$ and $E_{y}(x, z)$ are the orthogonal linearly polarized components of the field, $k_{x}$ and $k_{y}$ being their wave vector $\left(\Delta k=k_{x}-k_{y}\right) . \gamma$ is the nonlinear coefficient and $B=\chi_{x y y x} / \chi_{x x x x}$ is the polarization susceptibility ratio.

We then assume the propagation medium as isotropic, i.e., $k_{x}=k_{y}=k=2 \pi n_{0} / \lambda$. Thus $\Delta k=0$ and it is convenient to rewrite the CNLSE Eq. (1) using circularly polarized components $U, V=\left(E_{x} \pm \mathrm{i} E_{y}\right) / \sqrt{2}$. In this basis, the CNLSE are incoherently coupled and thus exhibit no energy exchange terms:

$$
\left\{\begin{array}{l}
\frac{\partial U}{\partial z}=\frac{i}{2 k} \frac{\partial^{2} U}{\partial x^{2}}+\mathrm{i} \gamma\left[\left((1-B)|U|^{2}+(1+B)|V|^{2}\right) U\right] \\
\frac{\partial V}{\partial z}=\frac{i}{2 k} \frac{\partial^{2} V}{\partial x^{2}}+\mathrm{i} \gamma\left[\left((1-B)|V|^{2}+(1+B)|U|^{2}\right) V\right]
\end{array}\right.
$$

The different terms in the right part of each equation represents, respectively, the effect of linear diffraction, SPM and XPM. We consider here, for the Kerr nonlinearity, the molecular reorientation effect of $\mathrm{CS}_{2}$, which prevails in the picosecond regime. In this case, $B=3 / 4$ and XPM is greater than SPM [16]. It has been demonstrated that given this parameter, SBI can manifest on a MVS [17].

Fig. 1 show a typical example of higher-order multimode vector solitary waves, namely, a three-soliton bound state. It consists in a vectorial combination of three in-phase solitons $(U)$ and three $\pi$-out-of-phase solitons $(V)$, propagating, respectively, in the fundamental and in the second-order modes of the total induced-waveguide. As initially demonstrated by Haelterman 
(a)

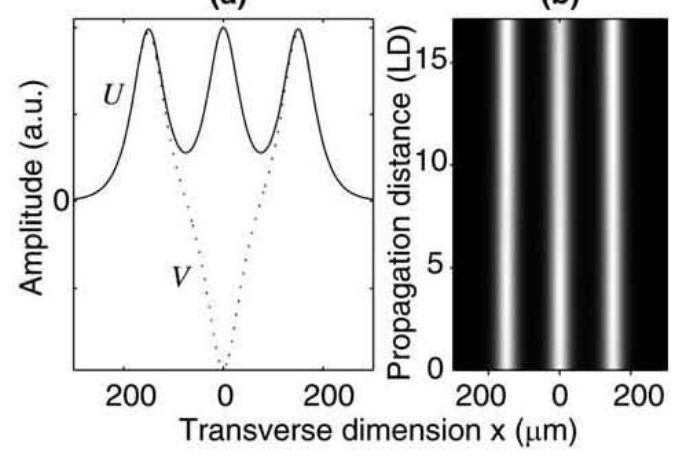

Fig. 1. (a) $(U, V)$ envelopes of the two-component three-hump vector soliton under study. (b) Numerical simulation showing stable propagation of the vector soliton over more than 15 diffraction lengths. The total input power is $2.1 \mathrm{~kW}$. One peakFWHM $x_{0}=50 \mu \mathrm{m}, \lambda=532 \mathrm{~nm}$, separation distance between peaks $B x=3 x_{0}$, mode height $e=9 \mu \mathrm{m}$.

and Sheppard [18], these waves are stationary solutions of Eq. (2) that branch from circularly polarized solitons. Their envelopes are therefore composed of two or more interacting solitons and obey the modal equation of a sech ${ }^{2}$ index profile multimode planar waveguide. A suitable arrangement of the wave parameters of the $U$ and $V$ fields indeed leads the attraction and repulsion forces between interacting solitons to be exactly compensated during propagation in the waveguide. Fig. 1(b) shows a numerical simulation of Eq. (2) with the initial field distribution of Fig. 1(a) that demonstrates an invariant propagation along more than 15 diffraction lengths. Here the diffraction length is defined by $L_{\mathrm{D}}=2.27 \Delta x^{2} n_{0} / \lambda$ where $\Delta x$ is the FWHM of one hump of the multihump soliton and $n_{0}$ the linear refractive index. The factor 2.27 is due to the fact that we consider here the FWHM rather than the 1/e full width. To get better insight, we have also plotted in Fig. 2(a) and (b), the nonlinear propagation within the Kerr medium of both $U$ and $V$ fields independently. We observe that the three in-phase solitons attract each other and finally merge into one circularly polarized soliton, whereas out-of-phase solitons repulse each other $[19,20]$ (Fig. 2(b)). The direct comparison between Figs. 1 and 2 clearly highlights that mutual trapping of the polarization components is necessary for the MVS to exist and to be stable.

However, when the XPM is larger than the SPM, it has been shown that MVS are under unstable equilibrium [17]. In particular, it has been recently reported that the bimodal vector soliton undergoes a sharp SBI [9]. As higher-order MVS rely also on an unstable equilibrium, it is interesting to study numerically how a small initial perturbation will act on the three-soliton bound state shown in Fig. 1. The results of these simulations are presented in Fig. 3 that shows several unstable behaviors of MVS for different small initial amplitude perturbations.

In Fig. 3(a), the MVS is initially perturbed by an increase of $20 \%$ in the amplitude of the central peak in $U$. One can observe a similar attraction dynamics as in Fig. 2(a) for three in-phase soliton, except that the propagation distance for merging is longer because of the influence of the $V$ field. The most interesting case is presented in Fig. 3(b) for which an asymmetric amplitude perturbations in the right-hand side of $V$ is introduced. As can be clearly seen in the output intensity profiles on Fig. 3(b), the solitary wave exhibits a SBI. The central and left-hand side of the MVS sharply merge while the right side propagates alone, slightly changing its direction due to repulsive effect. Though the initial amplitude perturbation was not so weak $(20 \%)$, the symmetry-breaking occurs after a rather long distance of propagation (20 diffraction lengths). Fig. 3(c) shows another kind of breaking dynamics where the perturbation

(a)

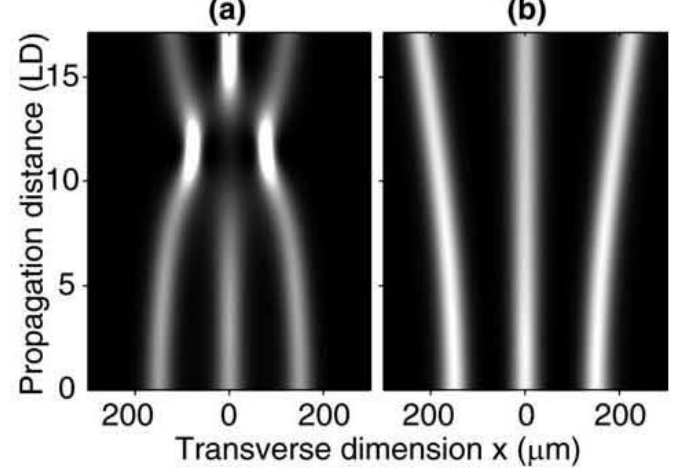

Fig. 2. Numerical simulations of the nonlinear propagation of (a) $U$ and (b) $V$ fields independently showing either attraction or repulsion of interacting vector solitons. Same parameters as in Fig. 1 and the input power is $4.3 \mathrm{~kW}$. 


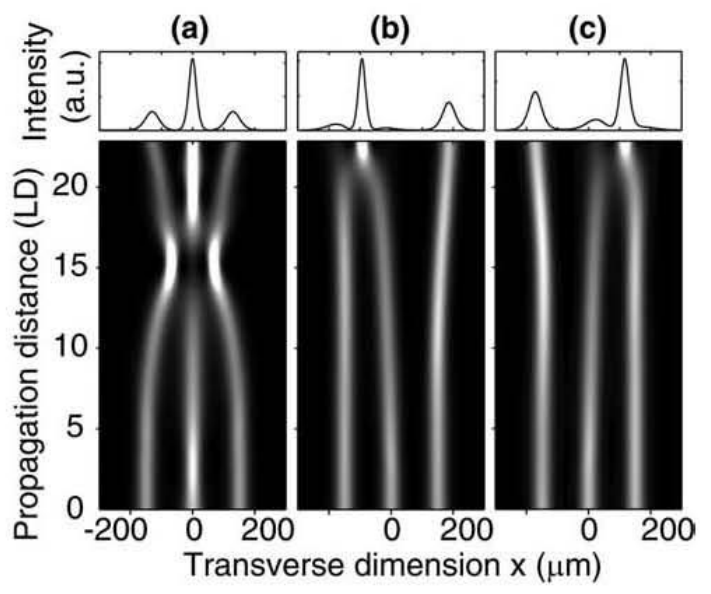

Fig. 3. Numerical simulations of the unstable propagation of the higher-order MVS of Fig. 1 under three different initial amplitude perturbations. Top: output intensity profiles.

is still asymmetric ( $10 \%$ increase of the $U$ amplitude and $5 \%$ increase of the $V$ amplitude on the left hand side). These results suggest that, on one hand, the direction of the SBI could be optically controlled for potential applications, for instance by launching a small signal to trigger off the SBI. On the other hand, different events can be obtained with SBI, each one of them corresponding to a useful guiding structure in view of potential applications for optical spatial switching and routing. Note that more complex functionalities could be generated using higher-order MVSs.

\section{Experiment}

The experiment is performed in a slab $\mathrm{CS}_{2}$ planar liquid waveguide using orthogonal circular polarization states of light as the two components of the MVS. The waveguide is made of a $10 \mu \mathrm{m}$ thick $\mathrm{CS}_{2}$ layer sandwiched between two SK 5 glass plates, with a refractive index difference $\Delta n=0.04$ [9]. The experimental setup for the two components of the MVS, depicted on Fig. 4, is based on a Michelson-type interferometer. A gaussian laser beam coming from a $10 \mathrm{~Hz}$ Q-switched, frequency-doubled, mode-locked Nd:YAG picosecond laser is split in two orthogonally polarized beams by means of a polarizing beam-splitter (PBS).

In one arm of the Michelson interferometer we have placed a silicon mirror, in which a $\lambda / 4$-thick and $300 \mu \mathrm{m}$ ridge has been etched. This mirror introduces the two $\pi$ phase-shifts necessary for shaping the $V$ component. A planar mirror is

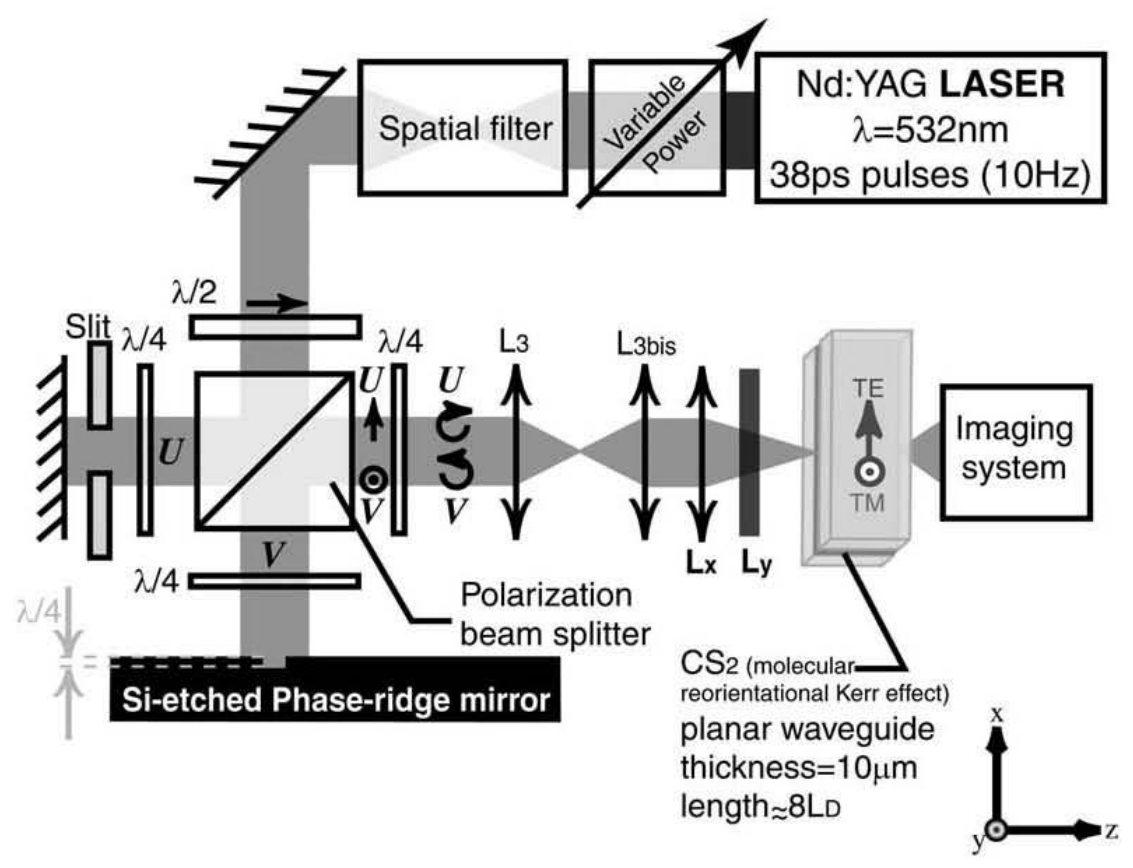

Fig. 4. Schematic of the experimental setup. 
placed in the other arm with a slit allowing adjustment of the width of the $U$ component. In both arms, a quarter-wave plate ensures total transmission through the PBS after reflection on the mirrors. Then, by passing through another quarterwave plate at the Michelson's output, the two resulting orthogonal polarizations are transformed into the required left- and right-handed circular polarizations. Both beams are reduced in size and launched inside the $3-\mathrm{cm}$ long $\mathrm{CS}_{2}$ waveguide by using a combination of spherical ( $L_{3}$ and $L_{3 \text { bis }}$ ) and cylindrical lenses $\left(L_{x}\right.$ and $\left.L_{y}\right)$. On the one hand, the couple of lenses ( $L_{3}$ and $L_{3 \text { bis }}$ ) is a $4 f$ setup that images the mirrors faces at the input end of the waveguide, on the other hand the crossed lenses $L_{x}$ and $L_{y}$ allow the soliton width to be tuned and a good coupling efficiency in the 10 $\mu \mathrm{m}$ planar waveguide, respectively. Output beams are finally analyzed by means of an imaging system and a single-shot CCD camera.

Fig. 5(a) shows the input beam intensity profiles in both polarization components imaged by removing the waveguide. As expected, the $V$-wave exhibits a two-node intensity distribution. The spacing between the left and right humps is $\delta x=121 \mu \mathrm{m}$ and then decreases to $114 \mu \mathrm{m}$ when combined with the gaussian-shaped $U$ component, as shows the total input profile in Fig. 5(b). The $U$ component, plotted in solid line, has a gaussian shape. Its profile is therefore different from the one used in numerical simulations but we can expect similar dynamical properties, as was previously demonstrated in [9]. Fig. 5(c) depicts the optical beam at the waveguide's output in the low-power linear regime when only diffraction occurs. Note that the $V$-wave diffracts more signif-

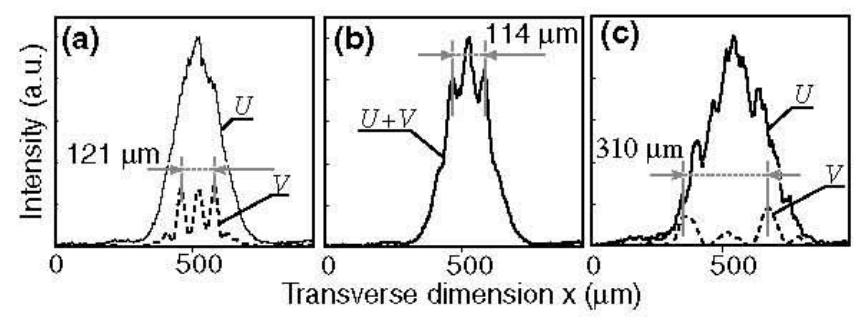

Fig. 5. (a) Input intensity profiles of $U$ and $V$; (b) total input intensity profile; (c) output intensity profiles in diffraction regime. icantly ( $\delta x=310 \mu \mathrm{m})$ than the $U$-wave because of the presence of higher spatial frequencies in its profile. The central hump of the $V$-wave decreases in intensity because of destructive interferences with both of the $\pi$-out-of-phase lateral humps.

When the nonlinear regime is reached, i.e., for a total measured peak power of $10 \mathrm{~kW}$, we observe that the output intensity distribution is strongly unstable from laser shot to shot, ranging from a three-hump to one single-hump distribution. Fig. 6(a) shows a typical event when the threehump beam is observed at the waveguide output. This means that the three-hump MVS remains stable during propagation, even though a noisy pedestal appears. This pedestal in mainly due to waveguide inhomogeneities and the diffraction of the leading and tailing edges of the picosecond pulse used in our experiment. We can note however that the spatial width is very close to the input one $(\delta x=102 \mu \mathrm{m})$. Fig. 6 (b) illustrates the $U$ and $V$ components launched separately without changing the input powers. As can be seen, the $U$-wave (solid line) exhibits a strong self-focusing effect while the $V$-wave still diffracts. The direct comparison with Fig. 6(a) therefore confirms the mutual trapping of both $U$ and $V$ components. These observations are in fairly good agreement with the theoretical predictions of Section 2 and the

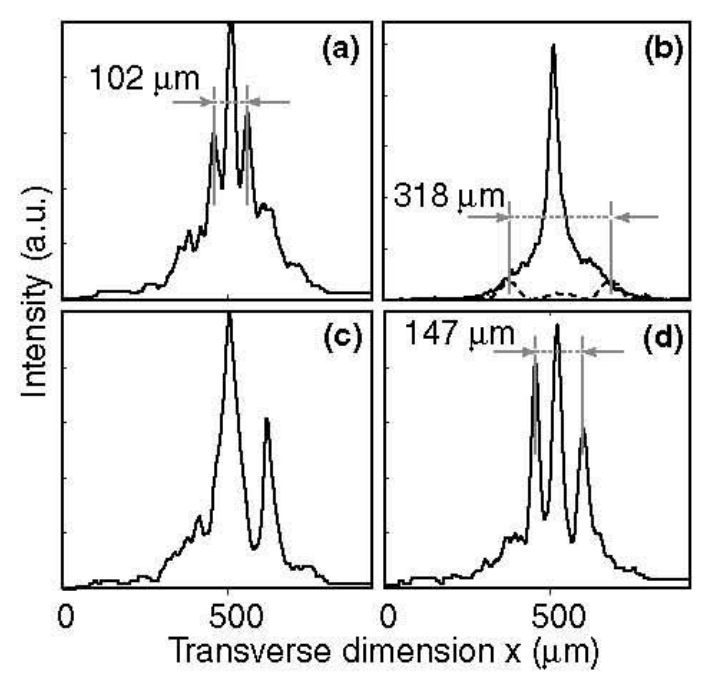

Fig. 6. (a) Output total intensity profile in the nonlinear regime for a peak power of $10 \mathrm{~kW}$. (b) Output intensity profile of $U$ and $V$ launched separately. (c, d) Intensity profiles showing SBI of the MVS. 
numerical simulation of Fig. 7 performed with experimental parameters. These simulations of the propagation of the profile depicted in Fig. 7(a) show several kinds of possible outputs: MVS in Fig. 7(b), symmetric evolution in Fig. 7(c) and SBI in Fig. 7(d). These events corresponding to different small input perturbations.

Fig. 6(c) illustrates another experimental output pattern that exhibits a clear-cut left SBI. Indeed, the beam is characterized by a two-hump intensity distribution with a strong central peak. Note that this pattern has been obtained without changing the experimental set-up, indicating that the laser beam noise is large enough to seed the SBI. A good qualitative agreement is obtained with the numerical simulation of Fig. 7(d) for which we have introduced an amplitude increase of $10 \%$ in the left hand side of $U$ and $V$ envelopes. Fig. 6(d) shows another output pattern observed where 3 peaks can be distinguished but where a slight

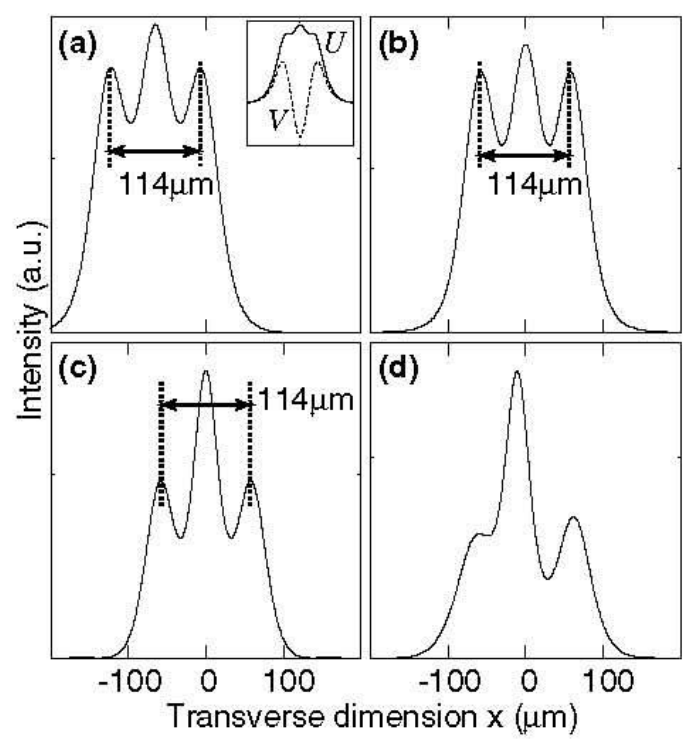

Fig. 7. Numerical simulations of the nonlinear propagation of the MVS under realistic conditions. (a) Input total intensity profile. Inset: ( $U, V)$ amplitude envelopes of the two-component vector soliton. (b) Numerical simulation showing stable propagation of the vector soliton over four diffraction lengths. (c) Numerical simulation showing unstable propagation. (d) Symmetry breaking of the same vector soliton perturbed by amplitude fluctuations. The total input power is approximatively of $1.6 \mathrm{~kW}$. FWHM $=50 \mu \mathrm{m}$ (for each sech shape), $\lambda=532 \mathrm{~nm}$, separation distance between peaks $B x=50 \mu \mathrm{m}$, mode width $e=9 \mu \mathrm{m}$. symmetry breaking appears. Experimentally, as many quasi-soliton bound-states and SBI-induced two-hump patterns are observed.

\section{Conclusion}

In conclusion, we have experimentally investigated the nonlinear propagation of a higher-order MVS, i.e., a three-solitons bound-state in a Kerr planar waveguide using the two opposite circular polarizations as the two components of the vector soliton. We have demonstrated the XPM-induced mutual trapping between the two components of this vector soliton. However, input noise of the laser field induces SBI on the vector soliton leading to a two-hump intensity distribution. These observations are in good agreement with numerical simulations of the CNLSE.

\section{Acknowledgments}

Authors thank Cyril Cambournac for helpful discussions and Denis Bitschene for technical assistance.

\section{References}

[1] M. Haelterman, A. Sheppard, A. Snyder, Opt. Lett. 18 (17) (1993) 1406.

[2] D. Christodoulides, R. Joseph, Opt. Lett. 13 (1) (1988) 53.

[3] Y. Silberberg, Y. Barad, Opt. Lett. 20 (3) (1995) 246.

[4] M. Mitchell, M. Segev, D. Christodoulides, Phys. Rev. Lett. 80 (21) (1998) 4657.

[5] Z. Chen, M. Acks, E. Ostrovskaya, Y. Kivshar, Opt. Lett. 25 (6) (2000) 417.

[6] D. Neshev, G. McCarthy, W. Krolikowski, E. Ostrovskaya, Y. Kivshar, G. Calvo, F. Agullo-Lopez, Opt. Lett. 26 (15) (2001) 1185.

[7] W. Krolikowski, E. Ostrovskaya, C. Weilnau, M. Geisser, G. McCarthy, Y. Kivshar, C. Denz, B. Luther-Davies, Phys. Rev. Lett. 85 (7) (2000) 1424

[8] Z. Chen, M. Segev, T. Coskun, D. Christodoulides, Y. Kivshar, V. Afanasjev, Opt. Lett. 21 (22) (1996) 1821.

[9] C. Cambournac, T. Sylvestre, H. Maillotte, B. Vanderlinden, P. Kockaert, P. Emplit, M. Haelterman, Phys. Rev. Lett. 89 (8) (2002) 083901.

[10] E. Ostrovskaya, Y. Kivshar, D. Skryabin, W. Firth, Phys. Rev. Lett. 83 (2) (1999) 296. 
[11] M. Haelterman, A. Sheppard, Phys. Lett. A 194 (1994) 191. [12] D. Pelinovsky, Y. Kivshar, Phys. Rev. E 62 (6) (2000) 8668.

[13] Z. Chen, Phys. Rev. E 57 (3) (1998) 3542.

[14] R. Malendevich, L. Friedrich, G. Stegeman, J. SotoCrespo, N. Akhmediev, J. Aitchison, J. Opt. Soc. Am. B 19 (4) (2002) 695.

[15] N. Akhmediev, A. Buryak, J. Soto-Crespo, Opt. Commun. 112 (1994) 278.
[16] R. Boyd, Nonlinear Optics, Academic Press, San Diego, 1992.

[17] P. Kockaert, M. Haelterman, J. Opt. Soc. Am. B 16 (5) (1999) 732.

[18] M. Haelterman, A. Sheppard, Phys. Rev. E 49 (4) (1994) 3376.

[19] J. Gordon, Opt. Lett. 8 (11) (1983) 596.

[20] J. Aitchison, A. Weiner, Y. Silberberg, D. Leaird, M. Oliver, J. Jackel, P. Smith, Opt. Lett. 16 (1) (1991) 15. 